\title{
3D Printed clothing house microorganisms
}

The wearable gut. An intestine-inspired tutu made with 58 meters of tubes filled with fluid is part of a 'wearable microbial factory' collection created by Massachusetts Institute of Technology Media Lab Professor Neri Oxman. The 3D design uses generative growth algorithms mimicking biological growth, and a Stratasys Objet Connex3 3D printer. The resulting tube, here filled with fluorescent liquid, varies in diameter from $1 \mathrm{~mm}$ to $2.5 \mathrm{~cm}$ and incorporates internal channels and pockets to support living organisms. The tube's transparency gradient-from clear to opaque-will one day allow photosynthetic cyanobacteria, for instance, to produce sucrose when exposed to light, which E. coli or yeast can convert to useful products such as new types of materials, drugs, food or scents. Collaborators include researchers at Harvard Medical School and the Harvard Wyss Institute for Biologically Inspired Engineering. See the video here: https://vimeo.com/131786000.

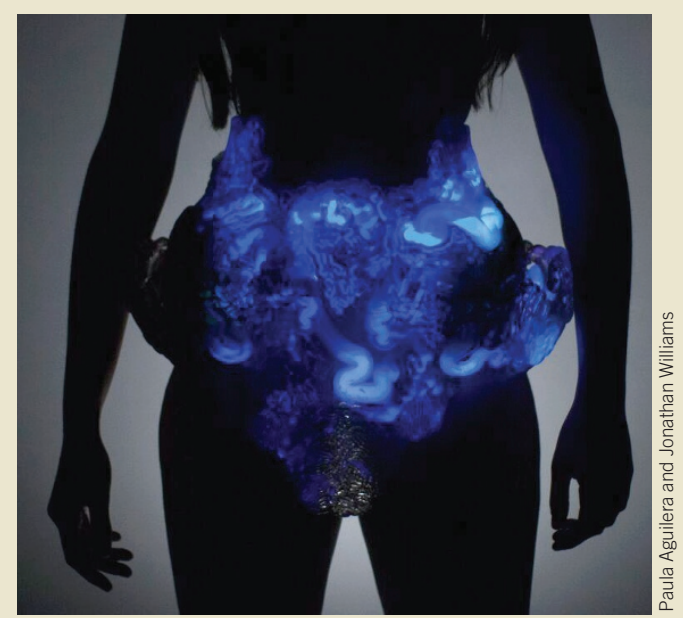

\section{Corrections}

In the August 2015 issue, in the article "Microbial cocktails join fecal transplants in IBD treatment trials," IBD was identified as irritable bowel disease, rather than inflammatory bowel disease. The error has been corrected in the HTML and PDF versions of the article.

In the same issue, in the article "Oxitec trials GM sterile moth to combat agricultural infestations," trials with a genetically modified moth were described as the first to be used in agriculture. The first were in Arizona testing a GM insect to control pink bollworm, a pest of cotton. The error has been corrected in the HTML and PDF versions of the article.

\section{Around the world in a month}

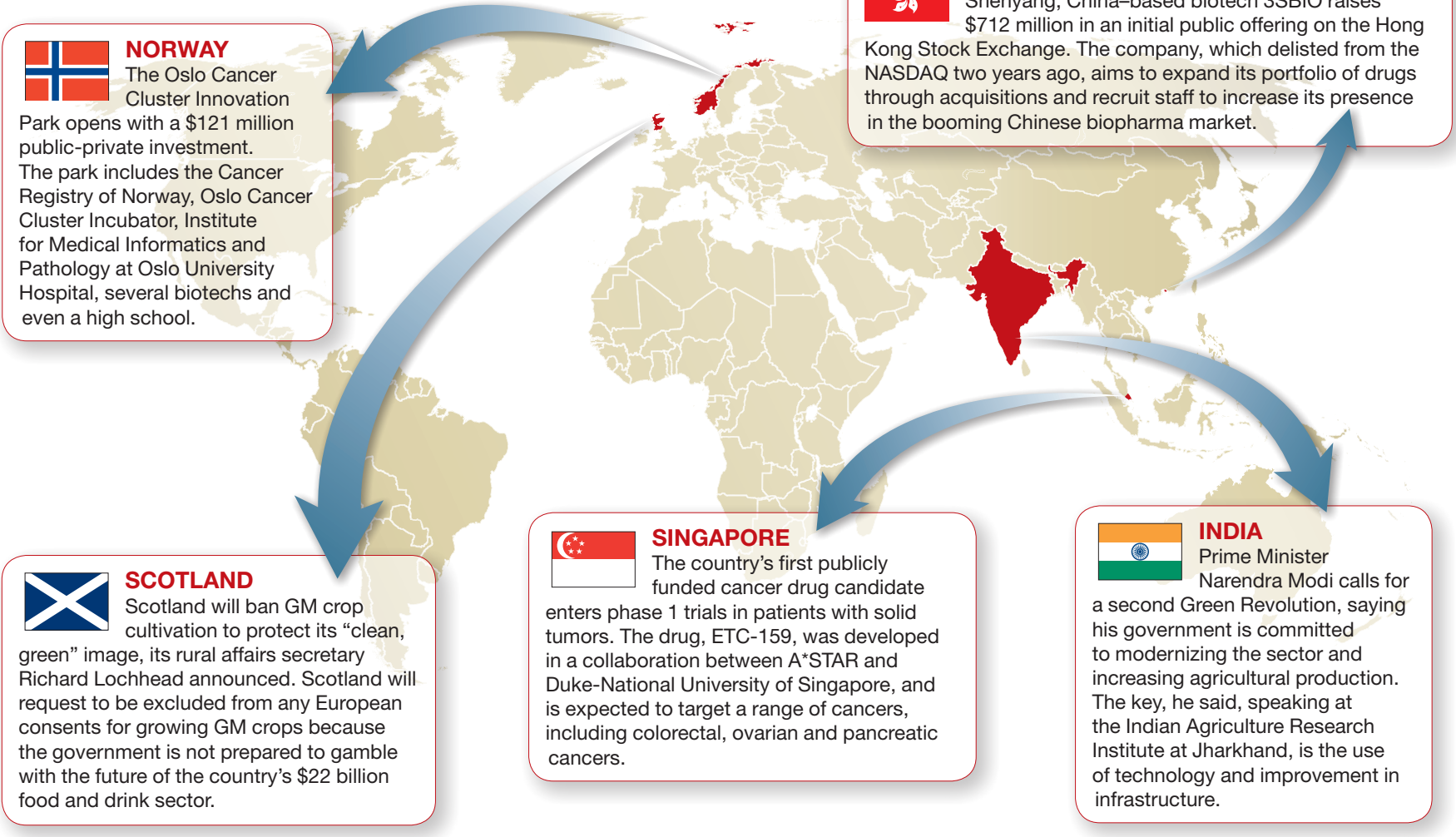

